Available online on 15.01.2018 at http://jddtonline.info
Journal of Drug Delivery and Therapeutics
Open Access to Pharmaceutical and Medical Research
(2011-17, publisher and licensee JDDT, This is an Open Access article which permits unrestricted non-
commercial use, provided the original work is properly cited

Open

Access

Research Article

\title{
EVALUATION OF DRUG PRESCRIPTION PATTERN USING WORLD HEALTH ORGANIZATION PRESCRIBING INDICATORS IN TIKUR ANBESSA SPECIALIZED HOSPITAL: A CROSS-SECTIONAL STUDY
}

\author{
Alemseged Beyene Berha ${ }^{1}$, Nathan Seyoum ${ }^{2}$ \\ ${ }^{1 *}$ Clinical Pharmacy Unit, Department of Pharmacology and Clinical Pharmacy, School of Pharmacy, College of Health Sciences, Addis \\ Ababa University, Addis Ababa, Ethiopia \\ ${ }^{2}$ Department of Pharmaceutics \& Social Pharmacy, School of Pharmacy, College of Health Sciences, Addis Ababa University, Addis Ababa, \\ Ethiopia
}

\section{ABSTRACT}

Background: Irrational use of antibiotics is a worldwide problem that contributes to dramatically increasing resistance and causes significant mortality, morbidity, and increased health care costs. In order to ensure the quality of health and medical care for patients and the community as a whole the proper use of medicines is an important element. Evaluation of drug use patterns with the WHO core drug use indicators is showing progressively more essential to promote rational drug use in developing countries. The main aim of this study was to assess the drug prescription pattern using WHO prescribing indicators at the Medical Outpatient Pharmacy in Tikur Ambessa Specialized Hospital (TASH).

Methods: A retrospective cross-sectional survey was designed to the claim data .A sample of 2000 prescriptions was selected using systematic random sampling to determine the prescribing pattern from a total of 6,462 prescriptions written by medical interns, residents and senior physicians for a one -year period from February 2015 to January 2016. The raw data was cleaned, entered and analyzed using SPSS version 21.0 of the computer software. The results were summarized using texts and tables.

Results: The average number of drugs per encounter was $2.84 \pm 1.58$ in the range of 1 to 9 . The total number of the drugs which were prescribed by generic name was $97.9 \%$. Of these, the total number of prescribed drugs which were included in the Ethiopian National Essential Medicine List was 99.3\%. The present study highlighted the under use of antibiotics which accounts $6.6 \%$ (378), with majority being Crystalline Penicillin $27.1 \%$ (94), followed by Amoxicillin 17.8\%(62) and Ciprofloxacin 14.1\% (49). A total of $8.4 \%$ (480) drugs were prescribed as injections.

Conclusion: On the basis of the finding of this study, the drug prescription pattern did not meet the WHO core drug use indicators criteria. It seems that there is a need for improvement of the prescribing patterns in the hospitals. Therefore, the federal ministry of health and drug regulatory body's commitment will be required to bring about changes in drug use policies, organize health systems and legislative structures as required in order to foster rational drug use in the country.

Keywords: Prescribing Indicators, Prescription, Rational Prescribing.

Article Info: Received 19 Dec, 2017; Review Completed 31 Dec, 2017; Accepted 10 Jan, 2018; Available online 15 Jan, 2018

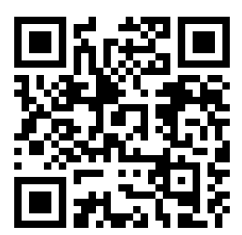

\section{Cite this article as:}

Berha $A B$, Seyoum N, Evaluation of drug prescription pattern using world health organization prescribing indicators in tikur anbessa specialized hospital: a cross-sectional study, Journal of Drug Delivery and Therapeutics. 2018; 8(1):74-80

DOI: http://dx.doi.org/10.22270/jddt.v8i1.1652

\section{*Address for Correspondence}

Alemseged Beyene (B.Pharm, MSc in Clinical Pharmacy), Coordinator, Clinical Pharmacy and Experiential Training Programs, Department of Pharmacology and Clinical Pharmacy, School of Pharmacy, College of Health Sciences, Addis Ababa University Addis Ababa, Ethiopia, Email: alemseged.beyene@aau.edu.et 


\section{BACKGROUND}

In order to ensure the quality of health and medical care for patients and the community as a whole the proper use of medicines is an important element. The World Health Organization (WHO) defined rational use of drugs as patients receiving medications appropriate to their clinical needs, in doses that meet their own individual requirements, for an adequate period of time and at the lowest cost to them and their community ${ }^{1}$.

It has been estimated that $50 \%$ or more medicine expenditure is being wasted through irrational prescribing, dispensing and patient use of medicine. Concurrent use of multiple medications by a patient result in drug induced disease like adverse drug reactions (ADR), which is reported to be as high as $28 \%$. Studies carried out in different areas of the globe, reveal divers drug use pattern. ${ }^{2}$. Irrational over use of medicines can stimulate inappropriate patient demand and lead to reduced access and attendance rates due to medicine stock outs and loss of patient confidence in health ${ }^{3}$.

Bad prescribing habits lead to ineffective and unsafe treatment, exacerbation or prolongation of illness, distress and harm to the patient, and higher costs. They also make the prescriber vulnerable to influences which can cause irrational prescribing ${ }^{4}$.

A method of measurement and a reference standard are useful to define the rational prescribing guide. The former is now available but the latter needs further development. To endorse rational prescribing by proven effective intervention in developed countries are treatment protocols based on wide consultation and consensus, properly introduced and with a possibility of feedback; face-to-face education focused on a particular prescribing problem in selected individuals; structured order forms; and focused educational campaigns 5 .

Prescribers can only treat patients in a rational way if they have access to an essential drugs list and essential drugs are available on a regular basis. ${ }^{2}$ Sometimes physicians say that they prescribe drugs to patients because patients demand them, so they are compelled to satisfy patients ${ }^{6}$. One of the roles of a professional body is to develop professional standards that are supportive, enabling and professionally challenging. There is a clear imperative for the providers of all pharmacy services to use professional standards to improve and develop services that are safe and put the needs of patients first ${ }^{7}$.WHO and the International Network of Rational Use of Drugs (INRUD) have developed a set of drug prescribing indicators to be used as measures of prescribing performance in primary care ${ }^{1}$. The drug use indicators are best understood as first line measures, intended to stimulate further questioning and to guide subsequent action ${ }^{8}$.

As much as possible choose a drug that is available, convenient and inexpensive to assure adherence and contain healthcare costs. The dose of drug varies according to a number of factors including age, weight, hepatic and renal functions, and severity of infection. The prescribing of the so-called "standard" dose in serious infections may result in failure of treatment or even death of the patient; therefore, it is important to prescribe a dose appropriate to the condition ${ }^{7}$.

Thus it is important that a check and balance system should, at least, review the prescriptions that were made out to patients so that the ultimate health care is being provided by the health care provider. The significance of the study, in large, is that it will help create awareness in order to implement a check and balance system so that health care providers can come as close to perfection as possible. Therefore, the aim of this study was to assess the prescribing pattern of physicians using World Health Organization (WHO) prescribing indicators at Medical Outpatient Pharmacy of Tikur Anbessa Specialized Hospital (TASH), Ethiopia.

\section{METHODS AND MATERIALS}

\section{Study Area and Period}

The study was conducted at Tikur Ambessa Specialized Hospital (TASH), Addis Ababa, Ethiopia. TASH was established in September 1974 and accommodates more than 600 beds, has more than 1700 medical and non-medical staff, offers inpatient, outpatient and emergency services. TASH serves about 250,000 patients per year in its outpatient department and about 24,000 in the in-patient and same number in the emergency departments. It is an important part of Ethiopia's health system and provides complex curative care and is considered as a last referral level curative care facility ${ }^{9}$.

The study was carried out at the medical outpatient pharmacy of TASH for a period of two months starting from March 22 to June 2, 2016 on prescriptions that dated from February 2015 to January 2016.

\section{Study Design}

A retrospective cross-sectional survey was conducted using WHO drug use indicators to evaluate the prescribing practice.

\section{Data Collection and Measurement}

This study was carried out in the outpatient pharmacy department of TASH, from February 2015 to January 2016. Prescriptions for the patients were spread throughout the year to reduce bias due to seasonal changes. A sample of 2000 prescriptions for the treatment of various diseases was selected using systematic random sampling to evaluate the prescribing patterns of the facility. Three was the sampling interval used to select the prescriptions for the study.

The World Health Organization prescribing indicator form was used as a standard data collection tool for evaluation of prescribing practices at health facilities and these include.

Indicator 1: It indicates the average number of drugs per prescription to measure the degree of polypharmacy. Average, calculated by dividing the total number of drug products prescribed, by the number of prescriptions surveyed. It is not relevant whether the patient actually received the drugs. 
Indicator 2: This is the percentage of drugs prescribed by generic name and measured the tendency to prescribe by generic name. Percentage, calculated by dividing the number of drugs prescribed by generic name by the total number of drugs prescribed, multiplied by 100 .

Indicator 3: This indicator describes the percentage of prescriptions with an antibiotic prescribed. The purpose is to measure the overall level of misused of antibiotic. Percentage, calculated by dividing the number of prescriptions in which antibiotics were prescribed, by the total number of prescriptions surveyed, multiplied by 100 .

Indicator 4: This pointer shows the percentage of prescriptions with an injection prescribed. It measured the general level of overused and costly form of injectable drug therapy. Percentage, calculated by dividing the number of prescriptions in which injections were prescribed, by the total number of prescriptions surveyed, multiplied by 100 .

Indicator 5: This determined percentage of drugs that were prescribed from essential drug list or formulary. It is helpful to measure the degree to which prescribing practices conform to Ethiopian National Essential Medicine List Fifth Edition. Percentage, calculated by dividing the number of drugs prescribed which are listed on the essential drugs list or local formulary by the total number of drugs prescribed, multiplied by 100 .

\section{Sampling and Sample Size}

The sampling method was systematic random sampling. The sample size was 2,000 prescriptions from a total of 6,462 prescriptions. All Prescriptions that were prescribing by physicians during the study period were included in the cross sectional survey.

\section{Source Population}

The Source population was all the prescriptions found in the medical outpatient pharmacy of Tikur Ambessa Specialized Hospital., Addis Ababa.

\section{Study Population}

The target study populations were all antibiotic and nonantibiotic prescriptions from the source population. This included all dosage forms that were prescribed by physicians.

\section{Inclusion Criteria}

All prescriptions that were prescribed by physicians during the study period were included.

\section{Exclusion Criteria}

The prescriptions that had characterized by illegible handwriting, absent written date, inpatient prescription, the prescription that has biological products and prescription that were written out of this hospital were excluded.

\section{Data Analysis}

After the data was entered \& cleaned carefully, all analyses were done using statistical package for social sciences (SPSS) version 21.0.Descriptive statistics including: frequency, percentage ,mean, standard deviation and range were used to summarize the prescribing indicators using the following formula adopted from the WHO's manual for prescribing indicators assessment. ${ }^{10}$. The results were summarized using tables.

\section{Data Quality Assurance}

The data was self-collected using WHO's manual for prescribing indicators assessment. Each and every prescription was seen analyzed thoroughly without inaptitude. Abstraction tool and data was checked for accuracy and completion. To maintain the quality of the data, a data collection checklist was pre-tested for its completeness for coverage of critical domains and on randomly selected $5 \%$ prescription.

Ethical approval

Ethical clearance to carry out this study was obtained from School of Pharmacy, College of Health Sciences, Addis Ababa University.

\section{RESULTS}

In this study, from a total of 6,462 prescriptions found at outpatient department of pharmacy in TASH that were written by medical interns, general practitioners, residents and senior physicians. We selected 2000 prescriptions using systematic random sampling to analyze the current drug prescribing practice at TASH and hence determine the trends in rational use of drugs in the institution.

The total number of medications summed to 5687 . The average number of drugs per encounter was $2.84 \pm 1.58$ with the range of 1 to 9 . Percentages of prescriptions which had only one drug were $8.4 \%$ (479) and the polypharmacy was $29.1 \%$ (1656). The total number of the drugs which were prescribed by generic name was $97.9 \%$ ( 5569). High percentages $99.3 \%$ (5648) of drugs were prescribed from the list of Ethiopian National Essential Medicine Fifth Edition. The percentage of encounters with an injection and prescribed antibiotics was $8.4 \%$ (480) and $6.6 \%$ (378) respectively. (See in Table 1). 
Table 1: Results of the study of drug-use through WHO prescribing indicators at Tikur Anbessa Specialized Hospital, Addis Ababa, Ethiopia, February 2015 to January $2016(n=2000)$.

\begin{tabular}{llll}
\hline Prescribing Indicator & $\begin{array}{l}\text { Number of drugs } \\
(\mathbf{N})\end{array}$ & $\begin{array}{l}\text { Percentage } \\
(\boldsymbol{\%})\end{array}$ & Standard \\
\hline $\begin{array}{l}\text { Number of drugs per encounter } \\
\text { (mean+SD) (range) }\end{array}$ & $2.84 \pm 1.584(1-9)$ & - & - \\
\hline Average Number of drugs per encounter & 5687 & 2.8 & $1.6-1.8$ \\
\hline Prescriptions with only one drug prescribed & 479 & $8.4 \%$ & $29.1 \%$ \\
\hline $\begin{array}{l}\text { Prescriptions with five or more } \\
\text { drugs prescribed (polypharmacy) }\end{array}$ & 1656 & & $13.4 \%-24.1 \%$ \\
\hline Prescriptions with injection drugs prescribed & 480 & $6.4 \%$ & $100 \%$ \\
\hline Prescriptions with antibiotics prescribed & 378 & $97.9 \%$ & $100 \%$ \\
\hline Drugs prescribed by generic name & 5569 & $99.3 \%$ & \\
\hline $\begin{array}{l}\text { Drugs included in the Ethiopian National Essential } \\
\text { Medicine }\end{array}$ & 5648 & & \\
\hline number of prescriptions sampled & & & \\
\hline
\end{tabular}

From the total of 5687 prescribed drugs, the present study highlighted the under use of antibiotics which accounts $6.6 \%$ (378), with majority being Crystalline
Penicillin $27.1 \%(94)$, followed by Amoxicillin $17.8 \%(62)$ and Ciprofloxacin $14.1 \%$ (49). (See in Table 2).

Table 2: Most common antibiotics prescribed at Tikur Anbessa Specialized Hospital, Addis Ababa, Ethiopia, February 2015 to January $2016(n=378)$.

\begin{tabular}{lll}
\hline Items prescribed & Frequency & Percentage (\%) \\
\hline Crystalline Penicillin & 113 & 29.9 \\
Amoxicillin & 61 & 16.1 \\
Ciprofloxacin & 48 & 12.7 \\
Metronidazole & 27 & 7.1 \\
Co-trimoxazole & 23 & 6.1 \\
Cloxacillin & 18 & 4.8 \\
Doxycycline & 16 & 4.2 \\
Azithromycin & 14 & 3.7 \\
Clarithromycin & 14 & 3.7 \\
Ceftriaxone & 13 & 3.4 \\
Norfloxacin & 12 & 3.2 \\
Cephalexin & 12 & 3.2 \\
Ceftazidime & 7 & 1.9 \\
Total & 378 & 100 \\
\hline
\end{tabular}

n: number of prescribed antibiotics

A total of $8.4 \%$ (480) drugs were prescribed as injections. From these, the most frequently prescribed injection was Crystalline Penicillin 112 (23.3\%), followed by NPH $19.37 \%(93)$ and Tramadol $16.87 \%$ $(81 \%)$ (See in Table 3$)$.

Table 3: Most commonly prescribed injection drugs at Tikur Anbessa Specialized Hospital, Addis Ababa, Ethiopia, February 2015 to January $2016(n=480)$.

\begin{tabular}{lll}
\hline Items prescribed & Frequency & Percentage (\%) \\
\hline Crystalline Penicillin & 112 & 23.3 \\
NPH Insulin & 93 & 19.4 \\
Tramadol & 81 & 16.8 \\
Warfarin & 79 & 16.5 \\
Diclofenac & 72 & 15.0 \\
Diazepam & 23 & 4.8 \\
Metronidazole & 10 & 2.1 \\
Cloxacillin & 5 & 1.1 \\
Ceftriaxone & 5 & 1.0 \\
Total & 480 & 100 \\
\hline
\end{tabular}

$\mathrm{n}$ : number of prescribed injections 
A total of 22 prescriptions were found to have exhibited polypharmacy in which the prescription containing antibiotics. The most frequently prescribed combined antibiotics were Amoxicillin and Crystalline Penicillin by $18.18 \%$ followed by Ciprofloxacin and Doxycycline by $13.63 \%$. Amoxicillin was the most frequent drug to have been prescribed in conjunction with other antibiotics. (See in Table 4).

Table 4: Number of prescribed antibiotics in a prescriptions with five or more drugs at Tikur Anbessa Specialized Hospital, Addis Ababa, Ethiopia, February 2015 to January 2016 ( $\mathrm{n}=22)$.

\begin{tabular}{lll}
\hline Items prescribed & number of prescription & Percentage (\%) \\
\hline Amoxicillin and Crystalline Penicillin & 4 & 18.2 \\
Ciprofloxacin and Doxycycline & 3 & 13.6 \\
Amoxicillin and Clarithromycin & 2 & 9.1 \\
Amoxicillin and Ciprofloxacin & 2 & 9.1 \\
Azithromycin and Crystalline Penicillin & 2 & 9.1 \\
Clarithromycin and Crystalline Penicillin & 2 & 9.1 \\
Amoxicillin and Ceftazidine & 1 & 4.5 \\
Amoxicillin and Co- trimoxazole & 1 & 4.5 \\
Amoxicillin and Doxycyline & 1 & 4.5 \\
Amoxicillin and Metronidazole & 1 & 4.5 \\
Ciprofloxacin and Co- trimoxazole & 1 & 4.5 \\
Crystalline Penicillin and Norfloxacin & 1 & 4.5 \\
Doxycycline and Metronidazole & 1 & 4.5 \\
\hline
\end{tabular}

n: number of polypharmacy prescriptions having antibiotics

\section{DISCUSSION}

The average number of drugs per prescription at the medical outpatient pharmacy in TASH was 2.84 . This is higher than the ideal which is $1.6-1.8^{11}$ and lower than reported by Lalan et.al (2012) in Maharashtra (west central India) is also $3.62{ }^{12}$. In addition, the finding of this study is also somewhat higher than that of previous studies like in Hawassa University Hospital which reported an average number of 1.9 drugs per encounter 13 and the average number of drugs per patient was 0.98 in Gondar, 1.8 in Bahir Dar and 2.2 in Debre Tabor hospitals ${ }^{14}$. The reason for this could be due to the higher prevalence of co-morbid conditions especially related to cardiovascular disorders. However, these values were close to the limit of two drugs per encounter recommended by WHO ${ }^{15}$ and similar to or lower than figures reported for other countries ${ }^{16,17}$. In addition , they were found to be in line with previous reports by Bharti et.al (2008) in India where the average number of drugs per prescription was $2.8^{18}$.

The percentage of drugs prescribed in generic name at the medical outpatient pharmacy in TASH was $97.9 \%$ which is acceptable compared to the ideal $100 \%{ }^{11}$. In a similar study conducted in Hawassa University Hopsital the percentage of drugs prescribed in generic name was $98.7 \%$ which is slightly higher than our finding ${ }^{13}$. Another drug prescribing pattern study conducted in three hospitals in north-west Ethiopia indicated prescribing by generic names was more or less uniform among the hospitals but generally lower compared to the finding in this study (Gondar Hospital, 72.6\%; Bahir Dar Hospital, 70.5\%; and Debre Tabor Hospital, 84.1\%) ${ }^{14}$. In contrary to this study, small portion of drugs was prescribed in generic name $5.13 \%{ }^{19}$.

A total of $8.4 \%$ (480) drugs prescribed were injectables at the medical outpatient pharmacy of TASH which is lower than the ideal range of $13.4 \%-24.1 \%{ }^{11}$ and much lower compared to reports from Hawassa University Hospital $(38.1 \%)^{13}$, Ayder Referral Hospital $(23.6 \%)^{20}$, and Debre Markos Referral Hospital $(48.36 \%){ }^{21}$. The reason behind the low values from TASH may be associated with the knowledge and attitude of general practitioners favoring more drug formulations like oral formulations with regards to having options of an underlying disorder. Another good reason may be the nature of the disease conditions that do not require emergency resolution since most ambulatory cases do not require parenteral interventions.

Pertaining to the injectable drugs, the most commonly prescribed injectable medications were Crystalline Penicillin (23.3\%), followed by NPH (19.37\%) and Tramadol $(16.87 \%)$ at TASH. This finding differs from the previous studies ${ }^{13}$ that reported Ampicillin (21.4\%), Cloxacillin (13.4\%), and Crystalline Penicillin (12.4\%).

Antibiotics are the most commonly prescribed class of drugs and have been reported to account for almost $50 \%$ of the pharmacy budget in hospitals ${ }^{22}$. It is also agreed that in many hospitals a very high percentage of antibiotics are inappropriately prescribed ${ }^{23}$. However, in this study, the percentage of antibiotic encounters was $6.64 \%$ (378) which is very low compared to the standard or ideal $(20 \%-26.8 \%)^{11}$ and values reported by many other studies $13,20,21,24-30$. This may be due to the burden of various co-morbid diseases mainly associated with cardiovascular disorders and the extent to which medications specific to the condition were prescribed. Since TASH usually deals with chronic diseases the medications mostly prescribed could be non-antibiotic by their nature ${ }^{31}$.

Most commonly prescribed antibiotics were Crystalline Penicillin (27.08\%), Amoxicillin (17.8\%), and Ciprofloxacin $(14.1 \%)$. This is somehow consistent with 
previous reports ${ }^{13,27}$. Among the prescribed drugs the ones that were included in the Ethiopian National Essential Medicine List was 5648 (99.3\%). This was found to be close to the ideal $100 \%$ (11); and strengthens the finding by Desalegn et.al (2013) ${ }^{13}$. Whereas, drugs prescribed from the formulary in four selected hospitals of West Ethiopia ranged from 73.5 to $100 \%{ }^{32}$.

Polypharmacy is often resulting from the inappropriate prescribing of more drugs than clinically necessary. Polypharmacy has been shown to result in drug- drug interaction, risk of fatal combined or synergistic medication adverse drug events (ADEs), medication nonadherence and hence patients leads to substantial worsening of disease, death and increased health care costs. To evaluate polypharmacy in this study, the average number of drugs per prescription was noted to be 2.8 showing the practice of overprescribing in TASH compared to the WHO recommend limit of less than 2 10

\section{CONCLUSION}

In general, the findings of this study are in line with what had been reported locally and globally. Prescription of drugs from essential medicines list and use of generic name drugs were found to be satisfactory. The prescribing patterns of antibiotics and injectable medications were low compared to the ideal or standard. On the contrary, the extent of polypharmacy was observed to be much higher than the recommended values and hence inappropriate. The best intervention (s) for improving polypharmacy involves an interprofessional approach that often includes a clinical pharmacist. Since majority of the cases handled in

\section{REFERENCES}

1. Mahalli AAE. WHO/INRUD drug prescribing indicators at primary health care centres in Eastern province, Saudi Arabia. Eastern Mediterranean Health Journal. 2012; 18(11):6.

2. Karande S, Sankhe P, Kulkarni M. Patterns of prescription and drug dispensing. Indian journal of pediatrics. 2005; 72(2):11721.

3. Hogerzeil H. Promoting the rational use of medicines. WHO Essential Drugs and Medicines Policy. 2002.

4. T. P. G. M. de Vries RHH, H. V. Hogerzeil, D. A. Fresle. Guide to Good Prescribing. Geneva, Switzerland.: WHO, 1994.

5. Hogerzeil HV. Promoting rational prescribing: an international perspective. British Journal of Clinical Pharmacy. 1995; 39:16.

6. Ntšekhe M, Hoohlo-Khotle N, Tlali M, Tjipura D. Antibiotic Prescribing Patterns at Six Hospitals in Lesotho. Strengthening Pharmaceutical Systems, Center for Pharmaceutical Management, Management Sciences for Health. 2011; 1:40.

7. ASTBURY M. Professional Standards for Hospital Pharmacy: Optimising Patient Outcomes from Medicines. Royal Pharmaceutical Society. 2014; 2.

8. Mrs M. Anker MPB-J, Dr. Bimo, Prof A. Chowdhury. How to Investigate Drug Use In Facilities: Selected Drug Use Indicators. WHO/DAP/931. 1993.
TASH are chronic diseases, the number of medications per prescription could be high.

Practical recommendation for policy makers and practitioner to monitor and improve the prescribing and consumption practices. Attempts should be made to provide reviewed and updated standard treatment guideline and on job training for all levels of health care within the rapid introduction of new drugs and advanced therapeutics, continuing education, and therapeutic audit. Good prescribing requires a sound and up to date knowledge pharmacology and applied therapeutics. Ethiopian clinical pharmacists also have a key role in rational drug use through patient education, provision of prescribing information and provision of pharmaceutical care in an outpatient setting.

\section{Competing Interests}

The authors declare that they have no competing interests.

\section{Authors' Contributions}

ABB identified the study area, designed the study, supervised data collection, did the analysis and reviewed the manuscript; NS coordinated the study, collected the data and prepared the manuscript. Both authors read and approved the final manuscript.

\section{Acknowledgment}

Authors sincerely thank Addis Ababa University, College of Health Sciences, School of Pharmacy, Department of Pharmacology and Clinical Pharmacy for facilitating this study. We also thank Tikur Anbessa Specialized Hospital pharmacy staff for their cooperation and making the prescription papers available.

9. Hospital TA. Tikur Ambessa Hospital. aaueduet/aaubeta/chs/tikur-anbessa-specialized hospital/background-of-tikur-anbessa-hospital/. 2015.

10. Organization WH. How to investigate drug use in health facilities: selected drug use indicators. 1993.

11. Isah AO R-DD, Quick J, Laing R, Mabadeje AFB. The development of standard values for the WHO drug use prescribing indicators.archiveswhoint/produc2004/rducd/ ICIUM_Posters/1a2_txthtm. 2016.

12. Lalan BK, Hiray R, Ghongane B. Drug prescription pattern of outpatients in a tertiary care teaching hospital in Maharashtra. Int J Pharm Bio Sci. 2012; 3(3):225-9.

13. Desalegn AA. Assessment of drug use pattern using WHO prescribing indicators at Hawassa University teaching and referral hospital, south Ethiopia: a cross-sectional study. Hawassa: Hawassa University; 2013.

14. Zeruesenay Desta TA, Asfawoseen Gebre-Yohannes, Alemayehu Worku. Drug prescribing patterns for outpatients in three hospitals in north-west Ethiopia. Ethiopian Journal for Health Development. 2002; 16(2):9.

15. Organization WH. How to investigate drug use in health facilities Selected drug use indicators. 1993. 
16. Guyon A.B. BA, Ahmed J.U., Ahmed A.U., Alam M.S., . A baseline survey on use of drugs at the primary health care level in Bangladesh. Bull World Health Organ. 1994; 72(2):265-71.

17. Nifkar S KA, Majdzadeh R, Abdollahi M, . Monitoring of National Drug Policy (NDP) and its standard indicators; conformity to decisions of the nation drug selecting committee in Iran. BMC Int Healt Human Rights. 2005; 5(5):5.

18. Bhartiy SS SM, Nandeshwar S, Tiwari SC. Pattern of prescribing practices in the Madhya Pradesh, India. Kathmandu University Medical Journal 2008; 6(21):55-9.

19. Sharma P, Kapoor B. Study of Prescribing Pattern for Rational Drug Theraphy. 2003.

20. Birhanu Demeke FM, Admassu Assen, Wondim Melkam, Solomon Abrha, Birhanetensay Masresha, Tigist Asefa. Evaluation of drugs utilization pattern using WHO prescribing indicators in Ayder Referral Hospital, Northern Ethiopia. International Journal of Pharma Sciences and Research (IJPSR). 2015; 6(2):342-7.

21. Tigistu Alemu Desse TCE. Assessment of drug use pattern using World Health Organization core drug use indicators at Debre Markos Referral Hospital, Northwest Ethiopia. International Journal of Innovative Pharmaceutical Sciences and Research. 2014; 2(7):1270-88.

22. Zaran F, Rybak M. Pharmacy-based antimicrobial-monitoring service. American Journal of Health-System Pharmacy. 1992; 49(7):1701-6.

23. Kunin CM. Evaluation of antibiotic usage: a comprehensive look at alternative approaches. Review of infectious diseases. 1981; 3(4):745-53.

24. Lenjisa JL, Fereja TH. A Retrospective Analysis of Prescribing Practices through WHO Prescribing Indicators at Four Selected Hospitals of West Ethiopia. Journal of Bioanalysis \& Biomedicine. 2014; 6(2):29.
25. Bayew T, Wubshet H, Zeryawkal E. Do Private Health Institutions Prescribe Drugs more Irratioinally than the Publics? A Year Long Comparative Evaluation of Prescribing Indecators, Wolkite Town, South West Ethiopia. Indian Journal of Pharmacy Practice. 2013; 6(2).

26. Organization WH. Assessment of the pharmaceutical sector in Ethiopia. 2003.

27. Karimi A, Haerizadeh M, Soleymani F, Haerizadeh M, Taheri F. Evaluation of medicine prescription pattern using World Health Organization prescribing indicators in Iran: A crosssectional study. Journal of research in pharmacy practice. 2014; 3(2):39.

28. Mulugeta T Angamo NTWaNJR. Assessment of Patterns of Drug use by using World Health Organization's Prescribing, Patient Care and Health facility indicators in Selected Health Facilities in Southwest Ethiopia. Journal of Applied Pharmaceutical Science. 2011; 1(7):62-6

29. Satish Kumar B.P. PG, Praveen Kumar, B. Anjaiah. A study on drug use pattern using WHO prescribing indicators in inpatients of Medicine Department in a rural teritiary care teaching hospital. Indo American Journal of Pharmaceutical Research. 2015; 5(5):2231.

30. Nirajan Bhattarai DB, Sajina Kusi, Kripa Adhikari and Sagar Basnet. Prescription pattern in various medical wards of Tribhuvan University Teaching Hospital under World Health Organization (WHO) prescription indicators. International Journal of Pharma Sciences 2014; 4(3):572-6.

31. Amha Gebremedhin DS, Tesgaye Aster, Aziza Shad and Marcia Landskroener,. Tikur Anbessa (Black Lion) Hospital Belgium: International Network for Cancer Treatment and Research; 2016 [cited 2016 July 23 2016].

32. Fereja JLLaTH. A Retrospective Analysis of Prescribing Practices through WHO Prescribing Indicators at Four Selected Hospitals of West Ethiopia. Journal of Bioanalysis and Biomedicine. 2014; 6(4):29-32. 